\title{
American Sign Language Recognition System: An Optimal Approach
}

\author{
Shivashankara $\mathbf{S}$ \\ Department of Computer Science \& Engineering, Sri Jayachamarajendra College of Engineering, Mysuru, India \\ Email: shivashankar.research@gmail.com \\ Srinath S \\ Department of Computer Science \& Engineering, Sri Jayachamarajendra College of Engineering, Mysuru, India \\ Email: srinath@sjce.ac.in
}

Received: 08 February 2018; Accepted: 17 May 2018; Published: 08 August 2018

\begin{abstract}
The Sign language is a visual language used by the people with the speech and hearing disabilities for communication in their daily conversation activities. It is completely an optical communication language through its native grammar, be unlike fundamentally from that of oral languages. In this research paper, presented an optimal approach, whose major objective is to accomplish the transliteration of 24 static sign language alphabets and numbers of American Sign Language into humanoid or machine decipherable English manuscript. Pre-processing operations of the signed input gesture are done in the first phase. In the next phase, the various region properties of pre-processed gesture image is computed. In the final phase, based on the properties calculated of earlier phase, the transliteration of signed gesture into text has been carried out. This paper also presents the statistical result evaluation with the comparative graphical depiction of existing techniques and proposed technique.
\end{abstract}

Index Terms-American Sign Language, Gesture Recognition, ASL Alphabets, ASL Numbers, Preprocessing, Region Properties.

\section{INTRODUCTION}

The sign language (SL) is made by specifications of hand and facial idioms to express their views and thoughts of speech and hearing disabled persons with the normal (speech and hearing) people. Most of the normal persons may not clearly understand the sign language. Therefore, there is a massive communication gap between the deaf communities with the general public. There is an inevitability of technology support for speech impairment people as human translators are highly impossible to patronage speech impaired persons in their daily activities in all the time. By the advancement in science and technology, we can think of designing an approach that can interpret gesture signs into humanoid or machine decipherable text. This smoothens the conversation between normal and impaired people [1].
There are more than 120 distinctive sign lingos are used by speech impaired community of various nations throughout the universe such as American Sign Language (ASL), Indian Sign Language, Australian Sign Language, Italian Sign Language, Srilankan Sign Language, and many more. Over and above 70 million people in the universe and about 10 million people in India are using sign language as their prime medium of communication.

ASL is most widely used SL in the world and fourth most usable linguistic in North America. Not only in United States, ASL is also used in Canada, Mexico, West Africa, and Asia. More than 20 other nations including Jamaica, Panama, Thai, Malaysia in which English is the major communication language uses ASL for their hearing impaired community communication. Nearly two million hard of hearing people of USA and Canada are using ASL as their primary basis of communication [2]. ASL is a broad as well as complicated language that usages signs made by actions of finger and hand cumulative by means of postures of the body and expressions of the face. As ASL is seen as precise and genuine language, it has plentiful variations, like other languages do, such as French and Spanish. ASL is an outstanding form of interaction and favorable to an enormous portion of the speech impairment population. Its foundation, existing conditions, prospect hopes, and global impact are quite amazing and eye-opening [3].

ASL provides a set of 26 gesture signs named as an American Manual Alphabet that can be cast-off to spell out many of the English words available. The 19 various hand shapes of ASL are cast-off to make 26 American Manual Alphabets. An identical hand shape with diverse orientations is used for ' $\mathrm{K}$ ' and ' $\mathrm{P}$ ' letters signs. In ASL, also offers a set of 10 numeric gestures to sign the numbers ' 0 ' to '9'. ASL doesn't comprises built-in ASL equivalents signs for accurate nouns and technical terms [4]. Along with ASL Alphabets and Numbers, there are thousands of hand and facial gesture signs are available to sign the various English words as well. The set of 26 gesture signs of English Alphabets (A-Z) and 10 Numbers (0-9) are shown in Fig. 1. 


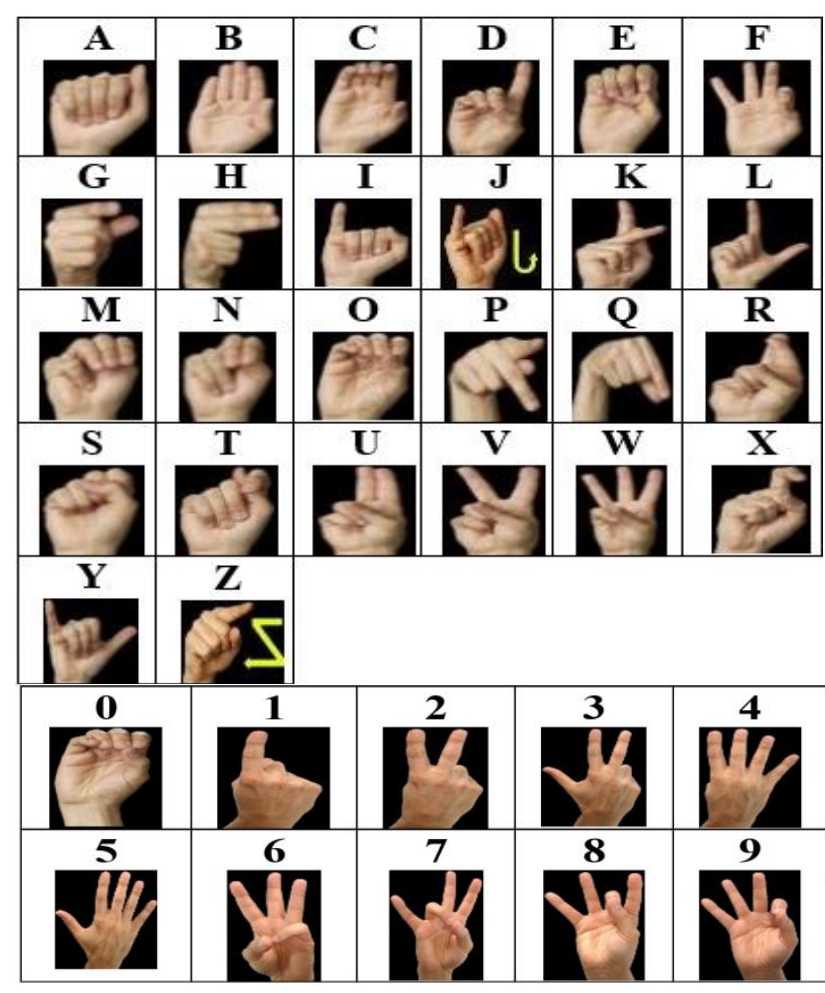

Fig.1. ASL Alphabets and Number gestures

\section{Motivation}

A good Sign Language Recognition (SLR) system can overcomes the barriers exists between the speech and hearing people with speaking society. The goal of SLR for developing systems and approaches for properly recognize the series of gestures and to know the meaning of the gestures [5]. Several methodologies to SLR imperfectly treat the difficulty as Gesture Recognition (GR). SL is a challenge that it is multi-channel; translating meaning via several manners at the same time. As the research of SL semantics are yet in its initial junctures, it is necessary to develop a novel, universal SLR system

SLR is a challenging and motivational task with respect to many constraints and factors. Some of the motivational factors for opting up the design of ASL Recognition System are discussed here.

SLR is a noticeable task due to its impact on humanoid society as the mute pupil facing huge communication gap with the speaking community. SLR is a challenging work because of its variation of hand gestures, facial expressions, body movements and many such variations and confines in this regard. A very less volume of work has been done in this lane to recognize the distance invariant, size invariant, rotation invariant, and race invariant ASL gestures with respect to background (plain and complex, uniform and non-uniform), location (indoor and outdoor), time (day and night), and light illumination (natural and artificial). Also, there is no noticeable work has been done predominantly for real time gesture recognition by considering various research disputes with respect to static and dynamic environment. There are huge amount of opportunities to carry out the research in recognizing ASL to make the communication easier between and mute and speaking community.

\section{DATA COLLECTION}

Data collection is a part of research work in all the research arenas comprising sciences, social science, technology, humanity, and business as well. In data collection procedure, methods may vary by discipline, the prominence on guaranteeing precise and authentic collection leftovers the identical. Irrespective of the discipline of the study, clear-cut collection of data is an essential stage to stabilizing the integrity of the research. The openly existing data collections are restricted both in mass and class. A ceremonial progression of data collection is vital as it ratifies that the data congregated are both definite and truthful. The ensuing conclusions be contingent on arguments signified in the outcomes are valid [6].

ASL static gestures and video gestures can be referred and analyzed from the website of American Sign Language University (ASLU) [7][8][9][10] for ASL Alphabets, Numbers, and Video Gestures. Creating large annotated ASL database for training and testing purpose is time consuming. However, in this research work, an effort has been put in creation of plenty of ASL Gestures and Video Gestures set of own in various background (plain and complex, uniform and non-uniform), location (indoor and outdoor), time (day and night), and light illumination (natural and artificial) by different signers for cognition (training) and recognition (testing) of American SLR system.

\section{RELATED WORK}

Limited efforts have been attempted in recognition of gestures made by finger spelling but with confines of recognition rate and time.

A classification approach for sign language recognition is proposed in [2]. This system recognizes $24 \mathrm{ASL}$ alphabets gestures and yields $86.67 \%$ of success rate. A real time ASL recognition system of 26 English alphabets with complex background and mixed lighting condition was presented using Edge Oriented Histogram [11] by using 10 Mega pixel web camera with maximum of 1 meter distance and this offers $88.26 \%$ of success rate. Matheesha Fernando et al., [12], presented a system for recognition. Among 50 ASL signed gestures, 5 signed gestures by every 10 signers (A, B, C, D, V Signed gestures) were considered. The total of 8 signs (A, B, C, $\mathrm{D}, \mathrm{L}, \mathrm{P}, \mathrm{V}$, and $\mathrm{Y}$ ) were warehoused and cast-off as masters containing the recognizing 5 and 3 other signed gestures. 12 signed gestures were unsuccessful to recognize evidently in usual background which provides a recognition rate of $76 \%$ using $\mathrm{Hu}$ moment classification. In [13], an ASL Recognition System presented using Self Organizing Map. A 7 different gestures (B, C, H, I, L, O, 
Y) of ASL of 10 different sets in real time environment with plain background and a set from the internet was used for testing and obtain the recognition rate of $92 \%$. In 2011, an ASL Recognition method is established which uses Cartesian Genetic Programming to identify the gestures of 26 ASL English alphabet [14]. This uses 26 gestures for training and a new set of 26 gestures recognition purposes. The recognition results of above $90 \%$ accurate.

In [15], new feature extraction techniques are proposed to recognition of static ASL signs of numbers 0 to 9 in plain background and obtained $74.69 \%, 82.92 \%, 87.94$, and $98.17 \%$ of recognition rates using Statistical Measures Technique, Orientation Histogram Technique, COHST (Combined Orientation Histogram and Statistical Technique), and Wavelet Features Technique respectively. An Open-Finger Distance Feature Measurement and Neural Network Classification Technique is used to recognize the ASL Numbers in [16], and obtained $92.09 \%$ of recognition rate.

In 2014, an ASL detection system has been designed [17] by detecting human skin color using HSV color model and edge detection technique with morphological operations. A total of 100 gestures are tested and $65 \%$ of the total gestures were recognized properly. Sruthi Upendran et al., proposed an ASL interpreter [18] which recognizes 24 static ASL alphabets in to textual form and further to speech using principle Component Analysis (PCA) and K-Nearest Neighbor (KNN) algorithm and obtained $77.29 \%$ recognition rate. In [19], a human computer interaction system for recognition of an ASL gesture ' $\mathrm{P}$ ' in a plain background using gray scale thresholding and edge detection technique. They have taken only one gesture for consideration.

An ASL recognition framework using MAdaline Neural Network classification technique has been designed [20] to recognize the standardized ASL consisting 26 American manual alphabets from A to Z. A novel technique to recognize the 26 static ASL gestures (A-Z) using polygon approximation and Douglas Peucker algorithm. This technique recognizes the open and closed finger gestures efficiently and results $79.92 \%$ accuracy [21]. The space, size, illumination and rotation invariant alphabet recognition approach [22] of ASL is evolved using SIFT algorithm. This approach is designed work well for both standard ASL database and homemade ASL database. A quantitative attempt is made to recognize real time gestures as well. A dynamic simple and complex background hand gesture recognition (HGR) integrated system is developed [23] using Gaussian and canny filters with flood fill algorithm. Alphabets A to L are considered for recognition and yield $84 \%$ and $58 \%$ accuracy in simple and complex background respectively.

Zhi-hua Chen et al., presents an HGR method using background subtraction and finger segmentation technique [24]. The rule classifier is used to foretell the gestures labels. Here, the performance is evaluated with 1300 hand gestures and yields better results. In [25], a hand gesture interpretation technique is employed using B spline curvature concept and geometric invariance method for recognizing 24 ASL alphabets gestures captured by web camera and offers satisfactory results. Recognition system of ASL finger spelling with phonological feature-based tandem models is developed in [26], using Hidden Morkov Model (HMM)-based baseline with Gaussian mixture observation distributions. Experiments are carried out in studio environment with 2 signers for finger spelling word recognition.

\section{PROPOSED WORK}

In this proposed work, an effort has been placed to recognize ASL Alphabets and Numbers, which mainly depends only on hand and fingers. The process of identifying ASL Alphabets and Numbers is distributed as preprocessing the input image, computation of the region properties of the preprocessed image, and transliteration from treated image to text.

\section{A. HSV Color Model}

HSV (Hue, Saturation, and Value) color model is a perceptual color model, used in separating an image luminance (V) from color facts. Opting an HSV color initiates with opting one of the existing hues, which is how most people narrate to color than does the RGB color space, and then regulating the shade and intensity value. HSV color space is used in the circumstances where color depiction acting a vital role. This color space allows person to specify the boundary of the skin pixels only in terms of hue and saturation. Illumination (V) provides brightness facts, it is usually ignored to lessen illumination dependency of skin color [17]. Therefore, in this proposed work, HSV color model is used to detect the skin region and mark the skin pixels in signed input gesture image.

\section{B. $\mathrm{YCbCr}$ Color Model}

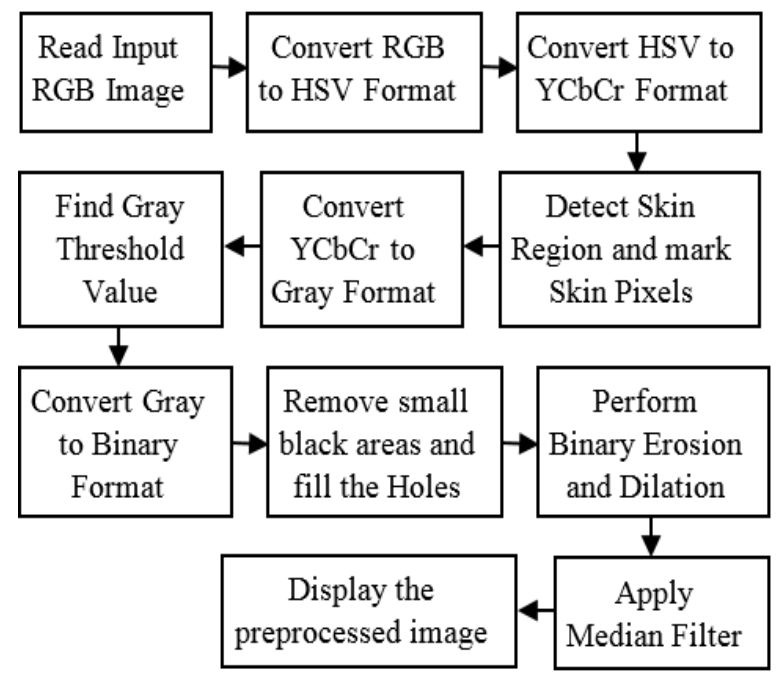

Fig.2. Block diagram of proposed method (Preprocessing Phase)

In order to optimize the functioning of skin color clustering, the present work uses $\mathrm{YCbCr}$ color space to construct a skin color model, as it is also known that, as the chrominance factors are almost self-governing of 
luminance factor in the space. There are non-linear relationships between chrominance $(\mathrm{Cb}, \mathrm{Cr})$ and luminance $(\mathrm{Y})$ of skin pixel color in the high and low luminance region. Luminance may vary depending on an individual's face because of ambient lighting [27]. Hence, the $\mathrm{YCbCr}$ Color model is very beneficial to optimize the performance of skin color clustering.

Fig. 2 illustrates the block diagram of proposed method of input gesture preprocessing.

\section{Preprocessing}

Step 1: Start

Step 2: Read an input RGB sign image.

Step 3: Convert an input image to HSV components.

Step 4: Convert $\mathrm{HSV}$ image form image to $\mathrm{YCbCr}$ components.

Step 5: Detect the Skin region of an image.

Step 6: Mark the Skin Pixels of an image.

Step 7: Convert YCbCr image to Gray components.

Step 8: Display the Gray image.

Step 9: Find the Gray threshold value using Otsu's method.

Step 10: Convert the Gray image to black and white components.

Step 11: Display the Black and White image.

Step 12: Remove the small black areas and fill the holes.

Step 13: Perform binary erosion and binary Dilation of the binary image.

Step 14: Perform Median Filter to reduce salt and pepper noise in an image.

Step 15: Display the image after erosion, dilation and median filtered.

Step 16: Display the final preprocessed image.

Step 17: Stop

Fig. 3 spectacles the block diagram of proposed method of Processing, and Transliteration process.

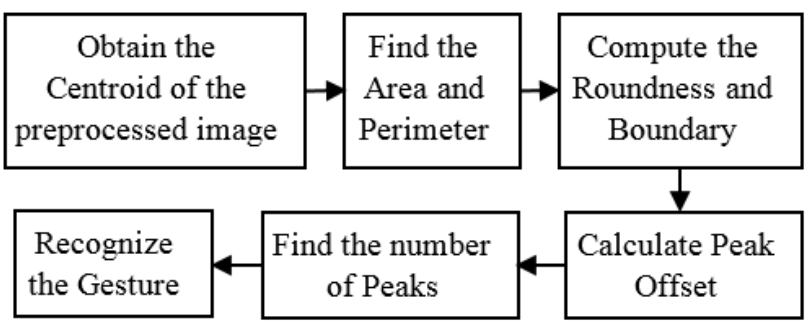

Fig.3. Block diagram of proposed method (Processing and Transliteration Phase)

\section{Processing and Transliteration}

Compute the properties of the region of the preprocessed image (all the shape measurements)

Step 1: Start

Step 2: Obtain the Centroid (Central mass of the region) of the preprocessed image.

Step 3: Find the Area (actual number of pixels in the region) and Perimeter (the distance around the boundary of the region) of an image.

Step 4: Equation (1) is used to find the Roundness and the Boundary of the preprocessed image.

$$
R N D=\frac{(4 * \pi * \mathrm{ARE})}{P E R^{2}}
$$

Step 5: Equation (2) is used to compute the Peak Offset point from the Centroid.

$$
\text { pkoffset }=\operatorname{CEN}(:, 2)+0.9 *(\operatorname{CEN}(:, 2))
$$

Step 6: Find the number of Peaks.

Step 7: Recognize the Gestures using the value of the Roundness and number of Peaks obtained.

Step 8: Stop

\section{RESULTS AND DISCUSSION}

To test the proposed work, we have taken the ASL alphabet and numbers of occluded and non-occluded gestures of different signers.

The recognition rate of the ASL alphabets and numbers gestures are calculated using the equation 3 as follows.

$$
\operatorname{Re} c o g ._{-} r a t e=\frac{\sum n o ._{-} o f \_ \text {gestures_recognized }}{\sum n o ._{-} o f_{-} \text {gestures_tested }}
$$

Following are the various screen shots obtained from the proposed algorithm for non-occluded gestures of ASL Alphabets.

In Fig. 4, 'Read an Image' button receives the nonoccluded input image gesture from the database and place it on the input screen for further preprocessing operations.

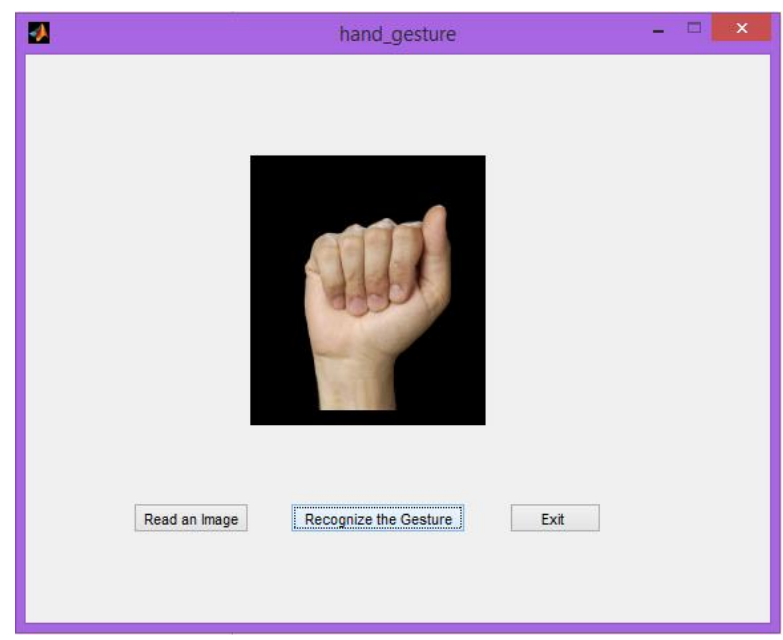

Fig.4. ASL Alphabet (Non-Occluded) Recognition Input

The $2^{\text {nd }}$ screen of the proposed algorithm is shown in Fig. 5, which displays the grayscale converted image of the input gesture, the binary (black and white) converted image. Thereafter an image of small black areas are removed and black holes are filled. It also displays the images which are Eroded, Dilated and Median Filtered as well. Finally the preprocessed image of the input gesture is displayed. 


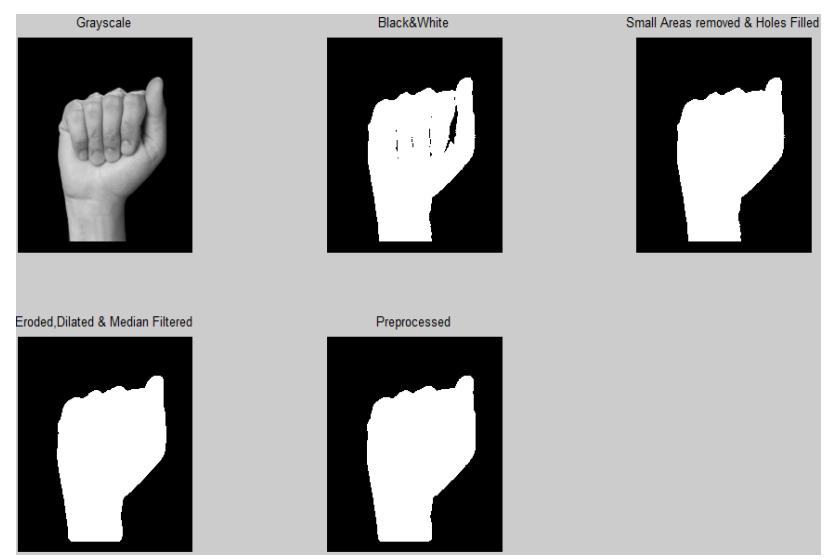

Fig.5. ASL Alphabet (Non-occluded) Recognition Process

The $3^{\text {rd }}$ and final screen of the proposed algorithm is shown in Fig. 6, which displays a non-occluded input image gesture (in this case) and preprocessed image with recognized alphabet along with the roundness value calculated for the respective gesture and number of peaks found. In recognition process, the input ASL gesture is recognized by comparing the roundness value calculated and number of peaks counted for the input gesture with the roundness value and number of peaks set of cognition (training) phase. Once the roundness value and the number of peaks of the input gestures are matched with range of roundness value and number peaks set, then the gesture will be recognized by displaying with the specified Alphabet.
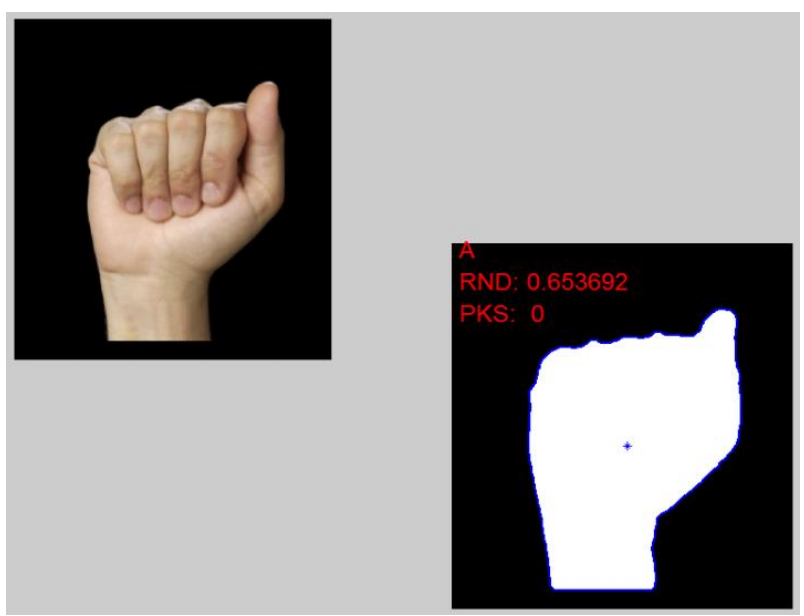

Fig.6. ASL Alphabet (Non Occluded) Recognition Output

In results evaluation of ASL Alphabets gestures, there are totally 6 ASL alphabet sample sets of 6 different signers with 6 various background colors were considered for testing. The ASL Alphabet-wise average recognition rate of 24 ASL alphabets, Set-wise average recognition rate of $6 \mathrm{ASL}$ alphabet sets, and overall recognition rate of all the ASL alphabet set (Set 1 - Set 6) are depicted in Table 1.
Table 1. Success Rate of ASL Alphabets

\begin{tabular}{|c|c|c|c|c|c|c|c|}
\hline Alphabet & $\begin{array}{c}\text { Set } \\
\mathbf{1}\end{array}$ & $\begin{array}{c}\text { Set } \\
\mathbf{2}\end{array}$ & $\begin{array}{c}\text { Set } \\
\mathbf{3}\end{array}$ & $\begin{array}{c}\text { Set } \\
\mathbf{4}\end{array}$ & $\begin{array}{c}\text { Set } \\
\mathbf{5}\end{array}$ & $\begin{array}{c}\text { Set } \\
\mathbf{6}\end{array}$ & $\begin{array}{c}\text { Success } \\
\text { Rate }\end{array}$ \\
\hline A & A & A & A & A & A & A & $\mathbf{1 0 0}$ \\
\hline B & B & B & B & B & B & B & $\mathbf{1 0 0}$ \\
\hline C & C & C & C & C & C & C & $\mathbf{1 0 0}$ \\
\hline D & D & D & D & D & D & D & $\mathbf{1 0 0}$ \\
\hline E & E & E & E & E & E & E & $\mathbf{1 0 0}$ \\
\hline F & F & F & F & F & F & F & $\mathbf{1 0 0}$ \\
\hline G & G & G & G & G & G & NI & $\mathbf{8 3 . 3 3}$ \\
\hline H & H & H & H & H & H & H & $\mathbf{1 0 0}$ \\
\hline I & I & I & I & I & I & I & $\mathbf{1 0 0}$ \\
\hline K & K & K & K & K & K & K & $\mathbf{1 0 0}$ \\
\hline L & L & L & L & L & L & L & $\mathbf{1 0 0}$ \\
\hline M & M & M & M & M & H & M & $\mathbf{8 3 . 3 3}$ \\
\hline N & N & N & N & N & NI & N & $\mathbf{8 3 . 3 3}$ \\
\hline O & O & O & O & O & O & O & $\mathbf{1 0 0}$ \\
\hline P & P & P & P & P & P & E & $\mathbf{8 3 . 3 3}$ \\
\hline Q & Q & Q & Q & Q & NI & NI & $\mathbf{6 6 . 6 6}$ \\
\hline R & R & R & R & R & R & R & $\mathbf{1 0 0}$ \\
\hline S & S & S & S & S & S & S & $\mathbf{1 0 0}$ \\
\hline T & T & T & T & T & T & T & $\mathbf{1 0 0}$ \\
\hline U & U & U & U & U & U & U & $\mathbf{1 0 0}$ \\
\hline V & V & V & V & V & T & V & $\mathbf{8 3 . 3 3}$ \\
\hline W & W & W & W & W & R & W & $\mathbf{8 3 . 3 3}$ \\
\hline X & X & X & X & X & M & X & $\mathbf{8 3 . 3 3}$ \\
\hline Y & Y & Y & Y & Y & B & Y & $\mathbf{8 3 . 3 3}$ \\
\hline Success & $\mathbf{1 0 0}$ & $\mathbf{1 0 0}$ & $\mathbf{1 0 0}$ & $\mathbf{1 0 0}$ & $\mathbf{7 0 . 8 3}$ & $\mathbf{8 7 . 5}$ & $\mathbf{9 3 . 0 5}$ \\
Rate & & & & & & \\
\hline & & & & & \\
\hline
\end{tabular}

(Note: In Table 1, ASL Alphabet $\mathbf{J}$ and $\mathrm{Z}$ have not been considered for recognition as they involve hand movements. Success Rate displayed is in \%)

Table 1 clearly shows that the Set 1 , Set 2 , Set 3 , and Set 4 provides $100 \%$ recognition rate. Here, as the Set 5 is an occluded gestures set, thus it gives the least success rate of $70.83 \%$ which is lesser compare to remaining 5 gestures sets as occlusion of the gesture degrades the recognition rate. In Set 5, the ASL alphabets gestures $\mathrm{N}$ and $\mathrm{Q}$ are not recognized and the alphabet gestures $\mathrm{M}, \mathrm{V}$, $\mathrm{W}, \mathrm{X}$, and $\mathrm{Y}$ are wrongly recognized. The Set 6 delivers moderately satisfactory result of $87.5 \%$ recognition rate. In Set 6, the ASL alphabet gestures $\mathrm{G}$ and $\mathrm{Q}$ were not recognized. The Overall Success rate in this test case is $93.05 \%$.

Fig. 7 illustrates the alphabet-wise average recognition rate of ASL gestures, where the ASL alphabet gesture Q offers $66.66 \%$ recognition rate, which is a least recognition rate comparing to remaining alphabets gestures. The ASL alphabets gestures G, M, N, P, V, W, $\mathrm{X}$, and $\mathrm{Y}$ provides the moderate recognition rate of $83.33 \%$, and rest of the ASL alphabets gestures yields an excellent recognition rate of $100 \%$ in all the gestures sets. 


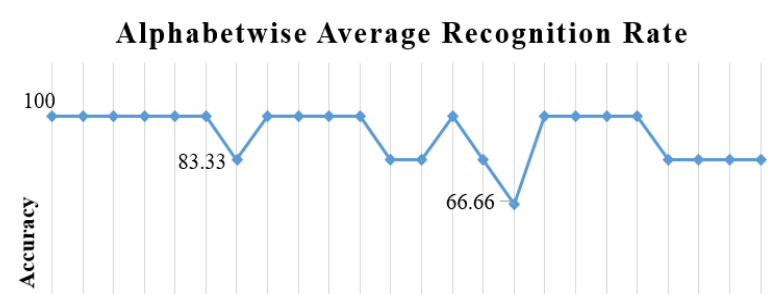

A B C D E F G H I K L M N O P Q R S T U V W X Y
ASLAlphabets

Fig.7. Alphabet-wise Average Recognition Rate.

The Set-wise average recognition rate of ASL alphabets gestures is shown in Fig. 8. It is noticed that, Set 1 , Set 2 , Set 3, and Set 4 provides $100 \%$ recognition rate for all the ASL alphabet gestures considered for testing. Here, as the Set 5 is an occluded gestures set, thus it gives the least success rate of $70.83 \%$ which is lesser compare to remaining 5 gestures sets. The Set 6 delivers moderately satisfactory result of $87.5 \%$ recognition rate.

\section{Setwise Average Recognition Rate}

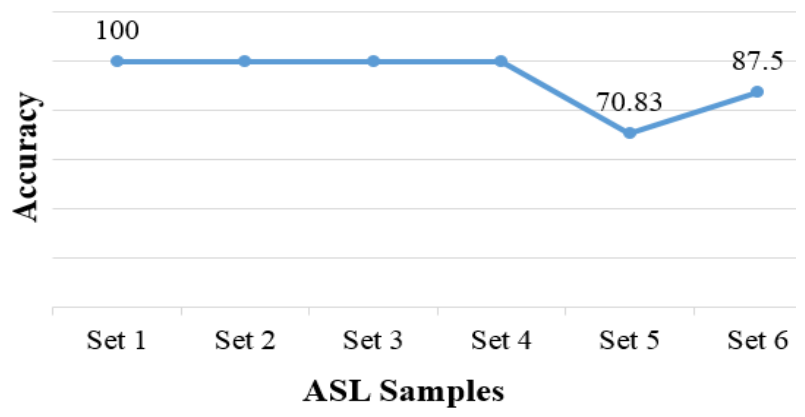

Fig.8. Set-wise Average Recognition Rate.

Following are the various screen shots obtained from the proposed algorithm for occluded gestures of ASL Alphabets.

Here, In Fig. 9, 'Read an Image' button receives the occluded input gestures from the database and place it on the input screen for further preprocessing operations.

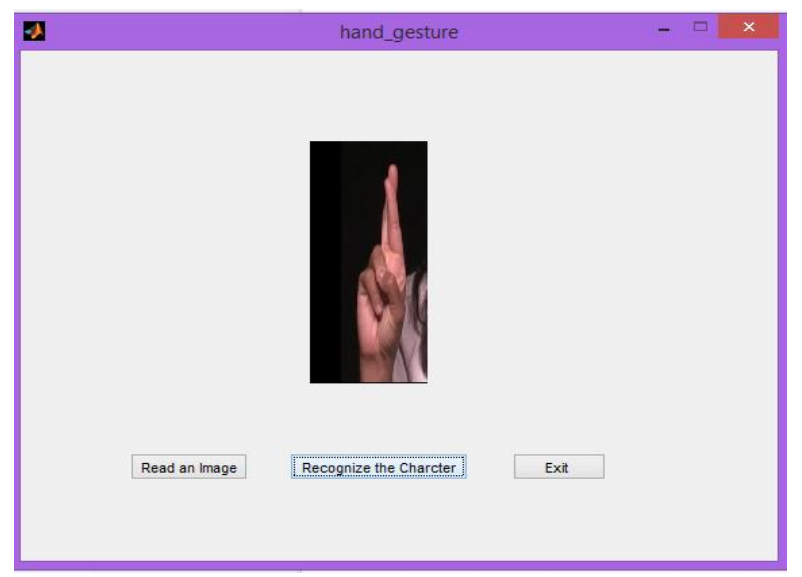

Fig.9. ASL Alphabet (Occluded) Recognition Input
The $2^{\text {nd }}$ screen of the proposed algorithm is shown in Fig. 10, which displays the grayscale converted image of the input gesture, the binary (black and white) converted image. Thereafter an image of small black areas are removed and black holes are filled. It also displays the images which are Eroded, Dilated and Median Filtered as well. Finally the preprocessed image of the input gesture is displayed.

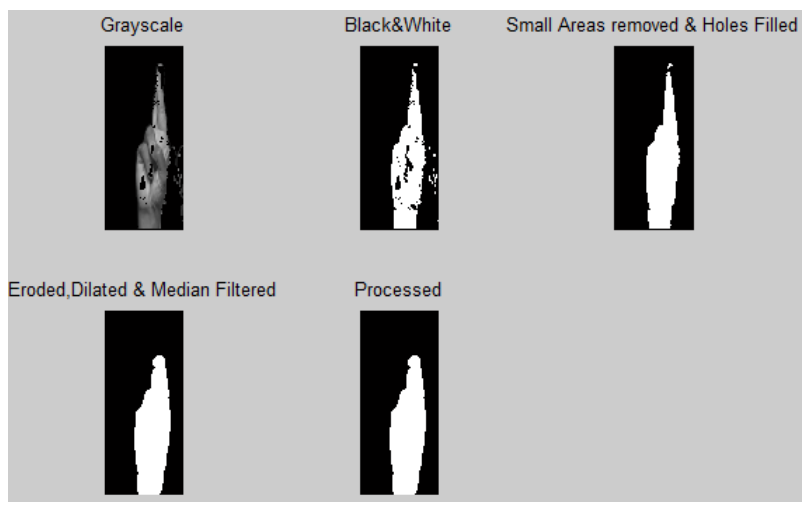

Fig.10. ASL Alphabet (Occluded) Recognition Process

The $3^{\text {rd }}$ and final screen of the proposed algorithm is shown in Fig. 11, which displays an occluded input image gesture (in this case) and preprocessed image with recognized alphabet along with the roundness value calculated for the respective gesture and number of peaks found.

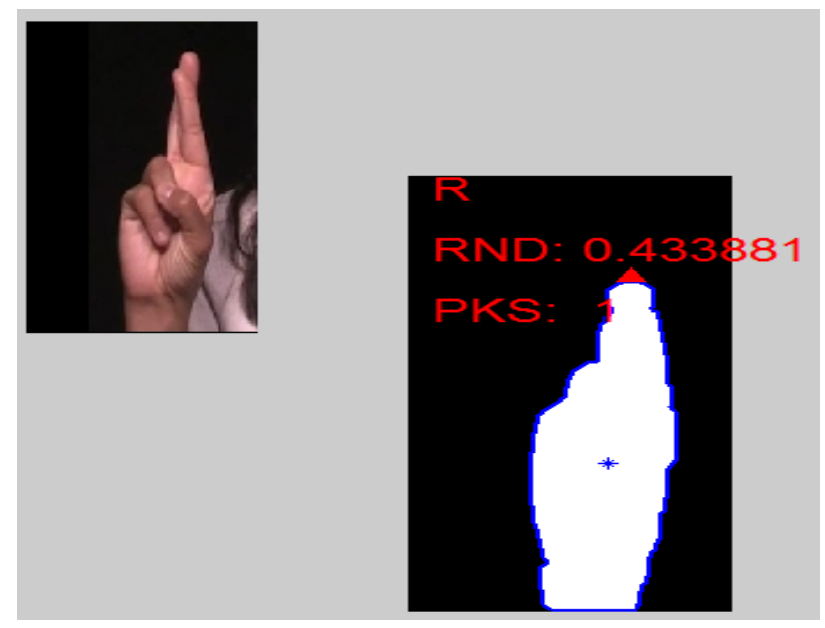

Fig.11. ASL Alphabet (Occluded) Recognition Output

Table 2 displays the Occluded and Non-occluded gestures of ASL Alphabets, which provides the average recognition rate of $70.83 \%$ (ASL alphabet gestures $M, N$, $\mathrm{Q}, \mathrm{V}, \mathrm{W}, \mathrm{X}$, and $\mathrm{Y}$ are not recognized) and $97.5 \%$ (ASL alphabet gestures $\mathrm{G}, \mathrm{P}$, and $\mathrm{Q}$ are not recognized in some data sets) respectively. Overall recognition result of nonoccluded gestures are much better than the recognition result of occluded gestures as occlusion of the gesture degrades the recognition rate. 
Table 2. Success Rate of Occluded and Non-occluded gestures of ASL Alphabets.

\begin{tabular}{|c|c|c|}
\hline \multirow[b]{2}{*}{ Alphabets } & \multicolumn{2}{|c|}{ Average Success Rate (in \%) } \\
\hline & Occluded Gestures & $\begin{array}{l}\text { Non-Occluded } \\
\text { Gestures }\end{array}$ \\
\hline $\mathbf{A}$ & 100 & 100 \\
\hline B & 100 & 100 \\
\hline $\mathbf{C}$ & 100 & 100 \\
\hline $\mathbf{D}$ & 100 & 100 \\
\hline $\mathbf{E}$ & 100 & 100 \\
\hline $\mathbf{F}$ & 100 & 100 \\
\hline $\mathbf{G}$ & 100 & 80 \\
\hline $\mathbf{H}$ & 100 & 100 \\
\hline $\mathbf{I}$ & 100 & 100 \\
\hline $\mathbf{K}$ & 100 & 100 \\
\hline $\mathbf{L}$ & 100 & 100 \\
\hline $\mathbf{M}$ & 0 & 100 \\
\hline $\mathbf{N}$ & 0 & 100 \\
\hline $\mathbf{O}$ & 100 & 100 \\
\hline $\mathbf{P}$ & 100 & 80 \\
\hline $\mathbf{Q}$ & 0 & 80 \\
\hline $\mathbf{R}$ & 100 & 100 \\
\hline $\mathbf{S}$ & 100 & 100 \\
\hline $\mathbf{T}$ & 100 & 100 \\
\hline $\mathbf{U}$ & 100 & 100 \\
\hline $\mathbf{V}$ & 0 & 100 \\
\hline $\mathbf{W}$ & 0 & 100 \\
\hline $\mathbf{X}$ & 0 & 100 \\
\hline $\mathbf{Y}$ & 0 & 100 \\
\hline Success Rate & 70.83 & 97.5 \\
\hline
\end{tabular}

Fig. 12 depicts the average recognition rate of occluded and non-occluded gestures of ASL Alphabets. In non-occluded gestures of ASL alphabets, the alphabets $\mathrm{G}, \mathrm{P}$, and $\mathrm{Q}$ gives $80 \%$ recognition rate. Whereas rest of the alphabets gestures offers $100 \%$ recognition rate. In occluded gestures of ASL alphabets, the alphabets M, N, $\mathrm{Q}, \mathrm{V}, \mathrm{W}, \mathrm{X}$, and $\mathrm{Y}$ gives NIL $(0 \%)$ recognition rate as they have not recognized at all. However, remaining alphabets offers $100 \%$ recognition rate.

Overall, it is noticed that non-occluded ASL alphabet gestures yields an outstanding recognition accuracy comparing with occluded ASL alphabet gestures.

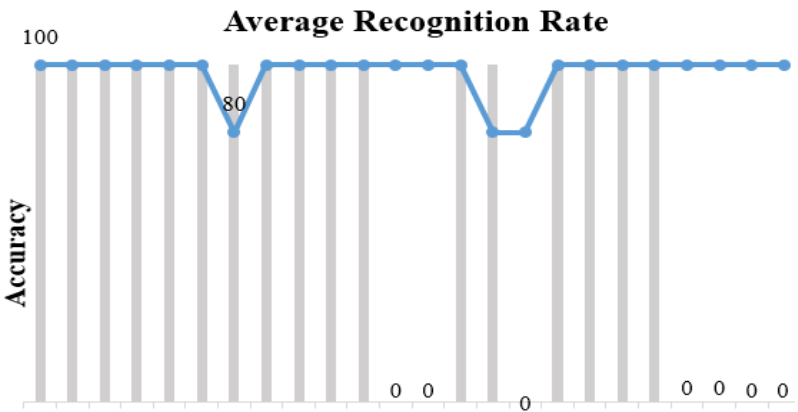

A B C D E F G H I K L M N O P Q

ASL Alphabets

Fig.12. Average Recognition Rate of Occluded and Non-occluded gestures of ASL Alphabets.

An Overall average recognition rate of Occluded and Non-occluded gestures of ASL Alphabets is shown in Fig. 13. Overall, it is noticed that non-occluded ASL alphabet gestures yields an outstanding recognition accuracy of
97.5\% for all 24 ASL alphabets comparing with 24 occluded ASL alphabet gestures of $70.83 \%$.

\section{Overall Average Recognition Rate}

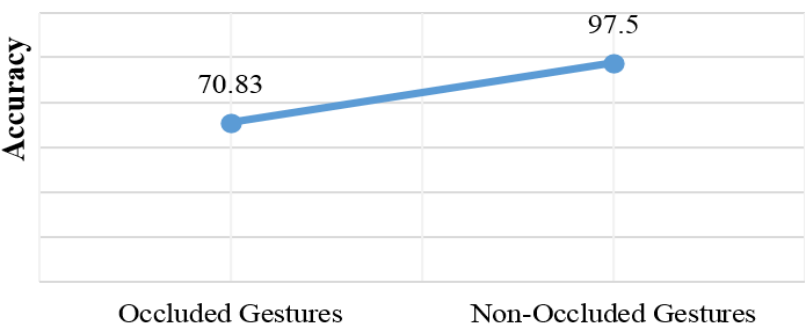

ASL Alphabets

Fig.13. Overall recognition rate of Occluded and Non-occluded ASL gestures.

Table 3. Recognition rates of various existing techniques with proposed technique for recognizing gestures of ASL Alphabets.

\begin{tabular}{|c|l|c|}
\hline $\begin{array}{c}\text { Reference } \\
\text { and Year }\end{array}$ & \multicolumn{1}{|c|}{ Technique Used } & $\begin{array}{c}\text { Recognition } \\
\text { Rate (in \%) }\end{array}$ \\
\hline$[2] 2017$ & Classification Approach & $86.67 \%$ \\
\hline$[11] 2016$ & Edge Oriented Histogram & $88.26 \%$ \\
\hline$[23] 2016$ & $\begin{array}{l}\text { Gaussian and canny filters with } \\
\text { flood fill algorithm }\end{array}$ & $71 \%$ \\
\hline$[18] 2014$ & PCA and KNN & $77.29 \%$ \\
\hline$[17] 2014$ & $\begin{array}{l}\text { HSV Color Model and Edge } \\
\text { detection }\end{array}$ & $65 \%$ \\
\hline$[12] 2013$ & Hu Moment Classification & $76 \%$ \\
\hline$[13] 2013$ & Self-Organizing Map & $92 \%$ \\
\hline$[14] 2011$ & Cartesian Genetic Programming & $79.92 \%$ \\
\hline$[21] 2011$ & $\begin{array}{l}\text { Polygon Approximation } \\
\text { Douglas - Peucker }\end{array}$ & $\mathbf{9 3 . 0 5 \%}$ \\
\hline $\mathbf{2 0 1 8}$ & Proposed Technique & \\
\hline
\end{tabular}

Table 3 shows that the recognition rate of proposed ASL alphabets gestures recognition technique and recognition rate of the existing traditional ASL alphabets gestures recognition techniques and with their recognition techniques used for ASL alphabets gestures recognition.

A comparative recognition rate of existing traditional ASL alphabets gestures recognition techniques and proposed ASL alphabets gestures recognition method for recognizing gestures of ASL Alphabets is illustrated in Fig. 14.

Table 3 and Fig. 14 clearly highlights the proposed ASL alphabets gestures recognition method yields the better ASL alphabets gestures recognition rate of $93.05 \%$ comparing with all the other existing traditional ASL alphabets gestures recognition techniques. It is also noticed that the proposed ASL alphabets gestures recognition method shows the huge recognition rate difference with the ASL alphabets gestures recognition technique with recognition rate of $65 \%$ which is implemented using HSV color Model and Edge detection system [17], and also with the ASL alphabets gestures recognition technique with the recognition rate of $71 \%$ which is implemented using Gaussian and Canny Filters with flood fill algorithm [23]. 


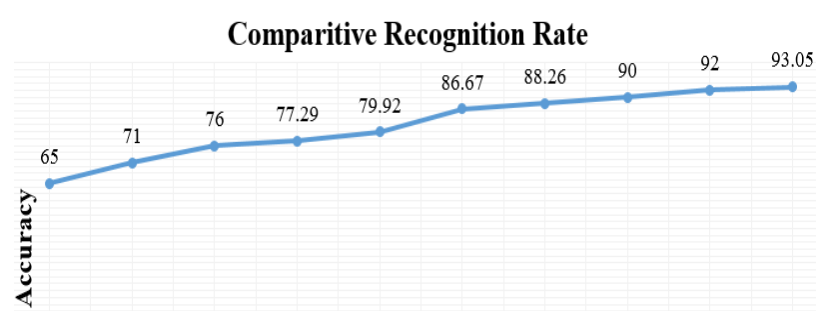

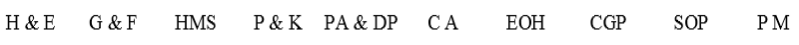
Techniques

Fig.14. A Comparative Success Rate of existing techniques and proposed methodology for recognizing gestures of ASL Alphabets.

(Note: In Fig. 14., abbreviations, H \& E: HSV Color Model and Edge detection, G \& F: Gaussian and Canny Filters with Flood Fill, HMS: Hu Moment Classification, S \& A: Support Vector Machines and Artificial Neural Networks, P \& K: PCA and KNN, PA \& DP: Polygon Approximation and Douglas - Peucker, CA: Classification Approach, EOH: Edge Oriented Histogram, CGP: Cartesian Genetic Programming, SOP: SelfOrganizing Maps, PM: Proposed Methodology)

Following are the various screen shots obtained from the proposed algorithm for non-occluded gestures of ASL Numbers.

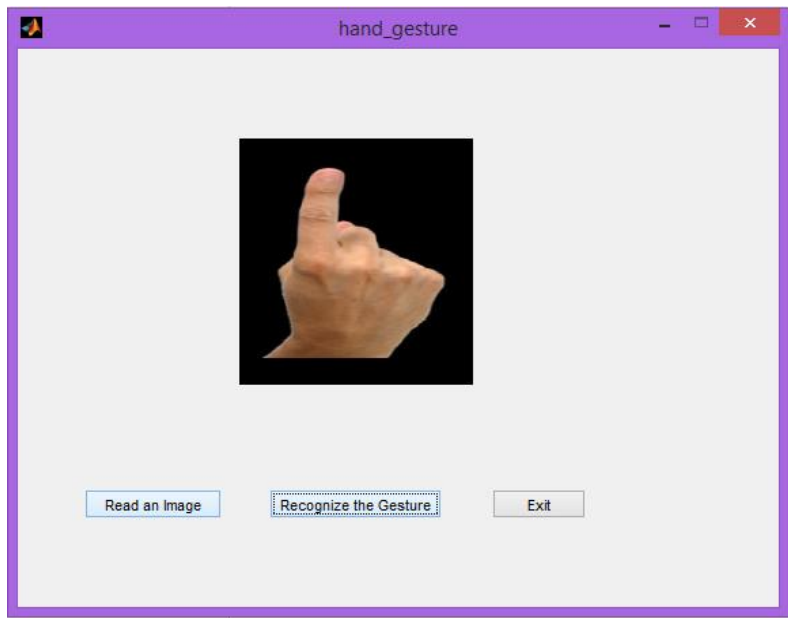

Fig.15. ASL Numbers (Non-Occluded) Recognition Input

Here, In Fig. 15, 'Read an Image' button receives the non-occluded input gestures from the database and place it on the input screen for further preprocessing operations.

The $2^{\text {nd }}$ screen of the proposed algorithm is shown in Fig. 16, which displays the grayscale converted image of the input gesture, the binary (black and white) converted image. Thereafter an image of small black areas are removed and black holes are filled. It also displays the images which are Eroded, Dilated and Median Filtered as well. Finally the preprocessed image of the input gesture is displayed.

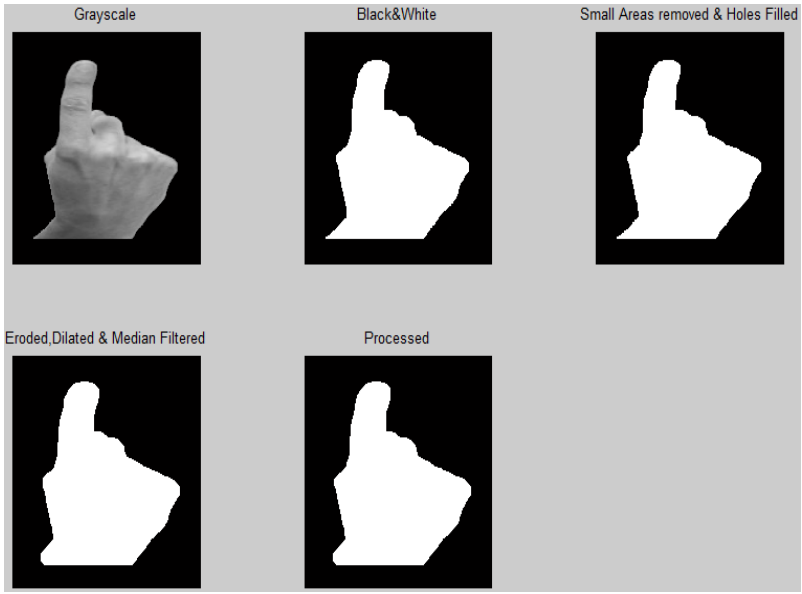

Fig.16. ASL Numbers (Non-occluded) Recognition Process

The $3^{\text {rd }}$ and final screen of the proposed algorithm is shown in Fig. 17, which displays the non-occluded input image gesture (in this case) and preprocessed image with recognized alphabet along with the roundness value calculated for the respective gesture and number of peaks found.
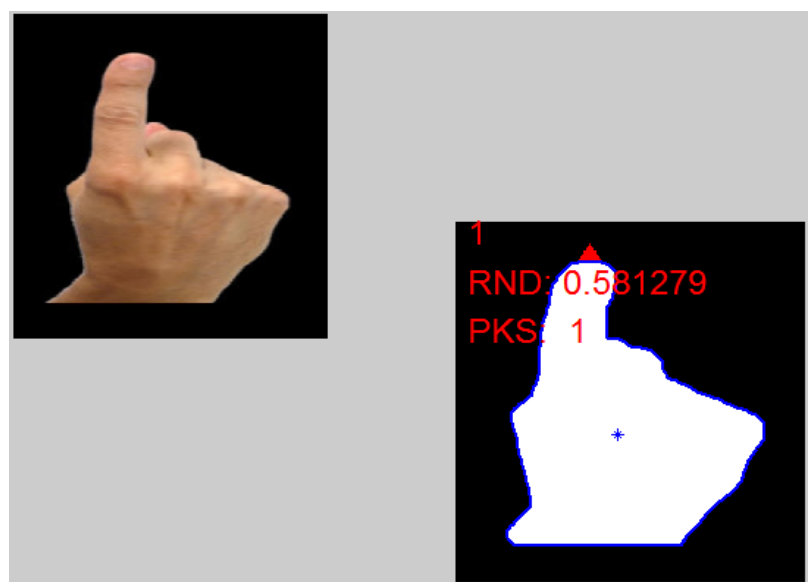

Fig.17. ASL Numbers (Non Occluded) Recognition Output

In results evaluation of ASL Numbers gestures, there are totally 6 ASL numbers sample sets of 6 different signers with 6 various background colors were considered for testing. The ASL Number-wise average recognition rate of 10 ASL Numbers, Set-wise average recognition rate of 6 ASL Numbers sets, and overall recognition rate of all the ASL Numbers gestures sets (Set 1 - Set 6) are depicted in Table 4.

Table 4 clearly shows that the Set 1 , Set 2 , Set 3 , and Set 4 provides $100 \%$ recognition rate. Here, as Set 5 and Set 6 are occluded gestures sets, thus these sets gives $80 \%$ and $90 \%$ success rate respectively. In Set 5, the ASL numbers gestures, the number 9 is not recognized and the number gesture 5 is wrongly recognized. In Set 6 , the ASL number gestures, the number gesture 8 is wrongly recognized. The Overall Success rate in this test case is $95 \%$. 
Table 4. Success Rate of ASL Numbers

\begin{tabular}{|c|c|c|c|c|c|c|c|}
\hline Numbers & $\begin{array}{c}\text { Set } \\
\mathbf{1}\end{array}$ & $\begin{array}{c}\text { Set } \\
\mathbf{2}\end{array}$ & $\begin{array}{c}\text { Set } \\
\mathbf{3}\end{array}$ & $\begin{array}{c}\text { Set } \\
\mathbf{4}\end{array}$ & $\begin{array}{c}\text { Set } \\
\mathbf{5}\end{array}$ & $\begin{array}{c}\text { Set } \\
\mathbf{6}\end{array}$ & $\begin{array}{c}\text { Success } \\
\text { Rate (\%) }\end{array}$ \\
\hline $\mathbf{0}$ & 0 & 0 & 0 & 0 & 0 & 0 & $\mathbf{1 0 0}$ \\
\hline $\mathbf{1}$ & 1 & 1 & 1 & 1 & 1 & 1 & $\mathbf{1 0 0}$ \\
\hline $\mathbf{2}$ & 2 & 2 & 2 & 2 & 2 & 2 & $\mathbf{1 0 0}$ \\
\hline $\mathbf{3}$ & 3 & 3 & 3 & 3 & 3 & 3 & $\mathbf{1 0 0}$ \\
\hline $\mathbf{4}$ & 4 & 4 & 4 & 4 & 4 & 4 & $\mathbf{1 0 0}$ \\
\hline $\mathbf{5}$ & 5 & 5 & 5 & 5 & $\mathbf{9}$ & 5 & $\mathbf{8 3 . 3 3}$ \\
\hline $\mathbf{6}$ & 6 & 6 & 6 & 6 & 6 & 6 & $\mathbf{1 0 0}$ \\
\hline $\mathbf{7}$ & 7 & 7 & 7 & 7 & 7 & 7 & $\mathbf{1 0 0}$ \\
\hline $\mathbf{8}$ & 8 & 8 & 8 & 8 & 8 & $\mathbf{3}$ & $\mathbf{8 3 . 3 3}$ \\
\hline $\mathbf{9}$ & 9 & 9 & 9 & 9 & $\mathbf{N I}$ & 9 & $\mathbf{8 3 . 3 3}$ \\
\hline $\begin{array}{c}\text { Success } \\
\text { Rate }\end{array}$ & $\mathbf{1 0 0}$ & $\mathbf{1 0 0}$ & $\mathbf{1 0 0}$ & $\mathbf{1 0 0}$ & $\mathbf{8 0}$ & $\mathbf{9 0}$ & $\mathbf{9 5}$ \\
\hline
\end{tabular}

Fig. 18 illustrates the Number-wise average recognition rate of ASL gestures, where the ASL Numbers 5, 8, and 9 offers $83.33 \%$ recognition rate, which is better recognition rate. Rest of the ASL numbers gestures yields excellent recognition rate of $100 \%$ in all the gestures set.

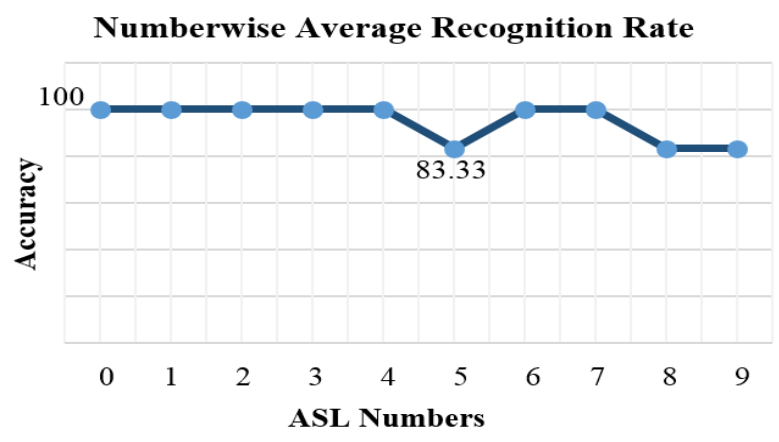

Fig.18. Number-wise Average Recognition Rate.

The Set-wise average recognition rate of ASL Numbers gestures is shown in Fig. 19. It is noticed that, Set 1 , Set 2, Set 3, and Set 4 provides $100 \%$ recognition rate for all the ASL numbers gestures considered for testing. Here, as the Set 5 and Set 6 are occluded gestures sets, thus these gestures sets gives the moderately satisfactory success rate of $80 \%$ and $90 \%$ respectively which are lesser compare to remaining 4 gestures sets.

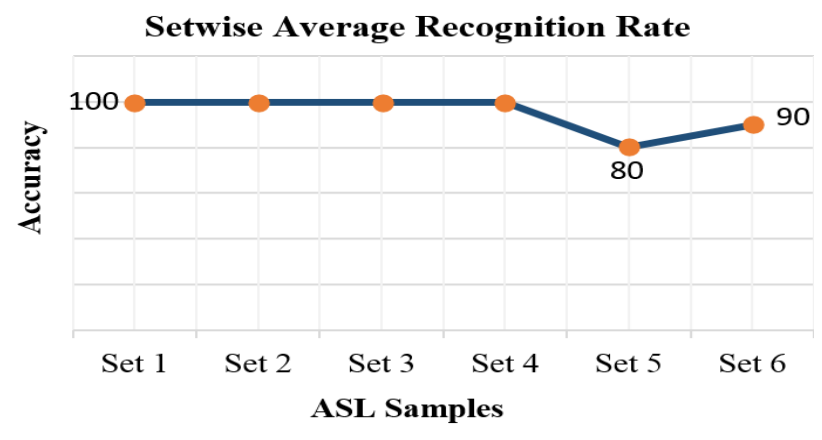

Fig.19. Set-wise Average Recognition Rate
Following are the various screen shots obtained from the proposed algorithm for occluded gestures of ASL Numbers.

Here, In Fig. 20, 'Read an Image' button receives the occluded input gestures from the database and place it on the input screen for further preprocessing operations.

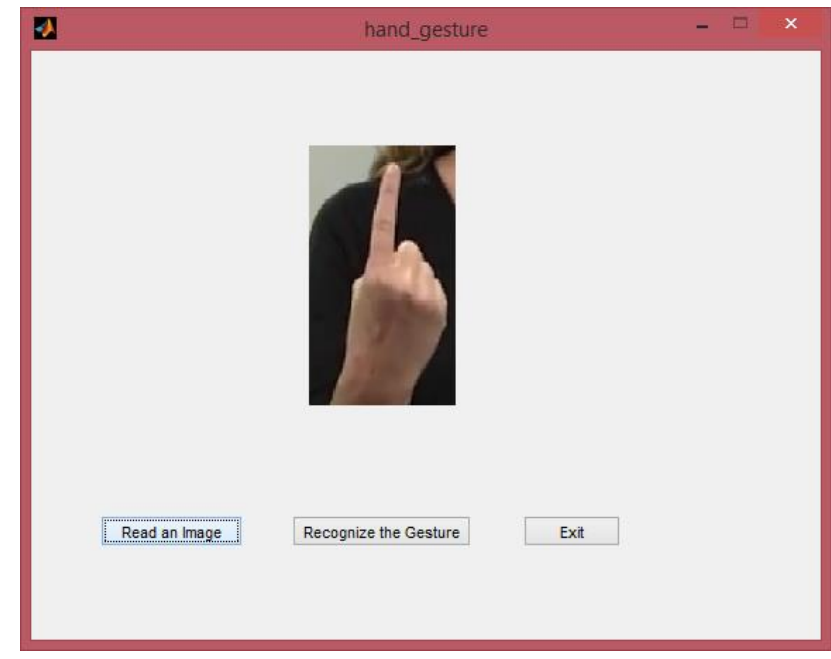

Fig.20. ASL Numbers (Occluded) Recognition Input

The $2^{\text {nd }}$ screen of the proposed algorithm is shown in Fig. 21, which displays the grayscale converted image of the input gesture, the binary (black and white) converted image. Thereafter an image of small black areas are removed and black holes are filled. It also displays the images which are Eroded, Dilated and Median Filtered as well. Finally the preprocessed image of the input gesture is displayed.

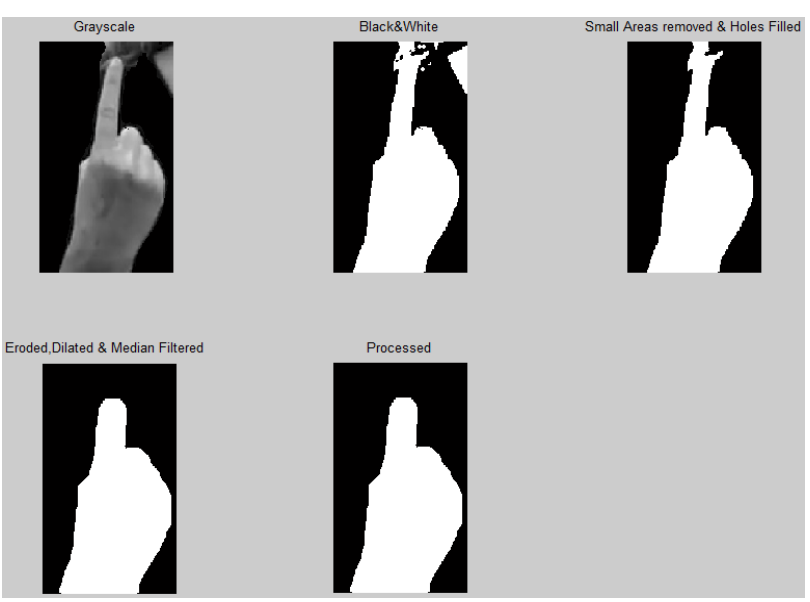

Fig. 21. ASL Numbers (Occluded) Recognition Process

The $3^{\text {rd }}$ and final screen of the proposed algorithm is shown in Fig. 22, which displays an occluded input image gesture (in this case) and preprocessed image with recognized alphabet along with the roundness value calculated for the respective gesture and number of peaks found. 


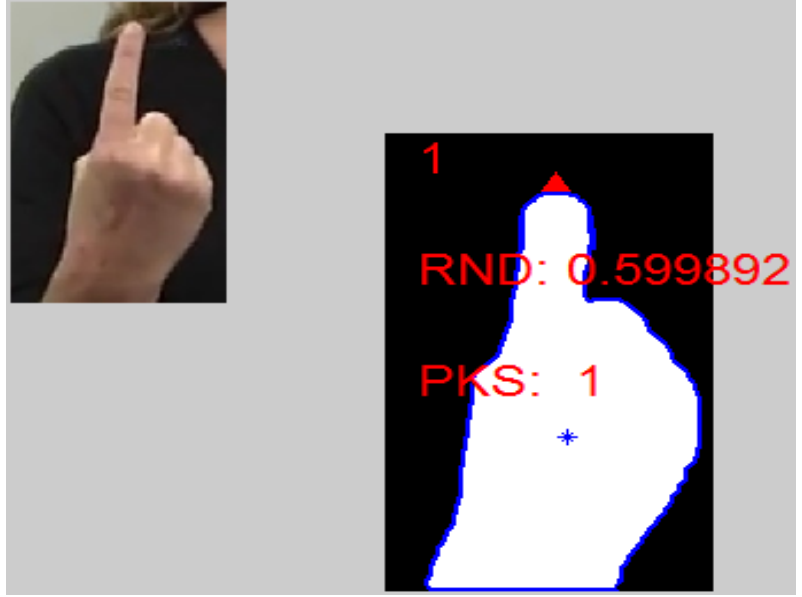

Fig.22. ASL Numbers (Occluded) Recognition Output

Table 5. Success Rate of Occluded and Non-occluded gestures of ASL Numbers

\begin{tabular}{|c|c|c|}
\hline \multirow{2}{*}{ Alphabets } & \multicolumn{2}{|c|}{ Average Success Rate (in \%) } \\
\cline { 2 - 3 } & Occluded Gestures & $\begin{array}{c}\text { Non-Occluded } \\
\text { Gestures }\end{array}$ \\
\hline $\mathbf{0}$ & 100 & 100 \\
\hline $\mathbf{1}$ & 100 & 100 \\
\hline $\mathbf{2}$ & 100 & 100 \\
\hline $\mathbf{3}$ & 100 & 100 \\
\hline $\mathbf{4}$ & 100 & 100 \\
\hline $\mathbf{5}$ & 50 & 100 \\
\hline $\mathbf{6}$ & 100 & 100 \\
\hline $\mathbf{7}$ & 100 & 100 \\
\hline $\mathbf{8}$ & 50 & 100 \\
\hline $\mathbf{9}$ & 50 & 100 \\
\hline Success Rate & $\mathbf{8 5}$ & $\mathbf{1 0 0}$ \\
\hline
\end{tabular}

Table 5 displays the Occluded and Non-occluded gestures of ASL Numbers, which provides the average recognition rate of $85 \%$ (ASL numbers gestures 5, 8, and 9 are wrongly / not recognized) and $100 \%$ respectively. Overall recognition result of non-occluded gestures sets provides an excellent recognition result than the recognition rate of occluded gestures as occlusion of the gestures degrades the recognition rate.

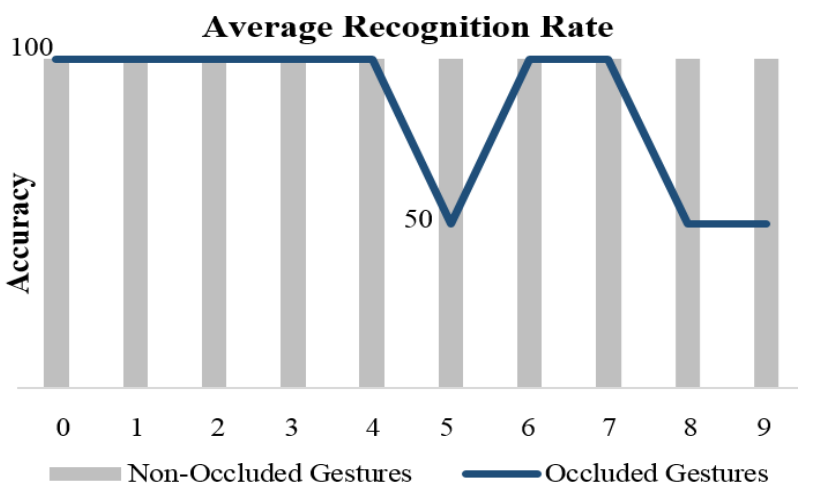

ASL Numbers

Fig.23. Average Recognition Rate of Occluded and Non-occluded Gestures of ASL Numbers.
Fig. 23 depicts the average recognition rate of occluded and non-occluded gestures of ASL Numbers. In non-occluded gestures of ASL numbers, the numbers 5, 8, and 9 gives the $50 \%$ recognition rate. Whereas rest of the numbers gestures offers $100 \%$ recognition rate but in occluded gestures of ASL numbers offers $100 \%$ recognition rate.

Overall, it is noticed that non-occluded ASL numbers gestures yields an outstanding recognition accuracy comparing with occluded ASL numbers alphabet gestures

An Overall average recognition rate of Occluded and Non-occluded gestures of ASL Numbers is shown in Fig. 24. Overall, it is noticed that non-occluded ASL numbers gestures yields an outstanding recognition accuracy of $100 \%$ for all 10 ASL alphabets (0-9) comparing with 10 occluded ASL numbers gestures of $85 \%$.

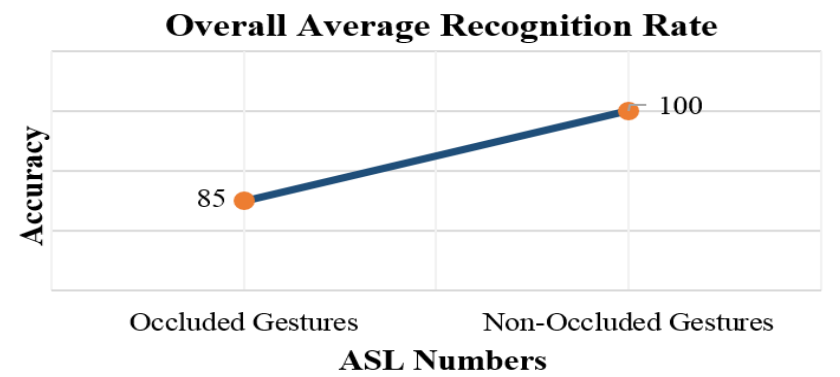

Fig.24. Overall recognition rate of Occluded and Non-occluded Gestures of ASL Numbers.

Table 6. Recognition Rates of various existing techniques with proposed technique for recognizing gestures of ASL Numbers.

\begin{tabular}{|c|l|c|}
\hline $\begin{array}{c}\text { Reference } \\
\text { and Year }\end{array}$ & \multicolumn{1}{|c|}{ Technique Used } & $\begin{array}{c}\text { Recognition } \\
\text { Rate (in \%) }\end{array}$ \\
\hline$[15] 2016$ & Statistical Measures & 74.69 \\
\hline$[15] 2016$ & Orientation Histogram & 82.92 \\
\hline$[15] 2016$ & $\begin{array}{l}\text { COHST (Combined Orientation } \\
\text { Histogram and Statistical) }\end{array}$ & 87.94 \\
\hline$[15] 2016$ & Wavelet Features & 98.17 \\
\hline$[16] 2015$ & $\begin{array}{l}\text { Open-Finger Distance Feature } \\
\text { Measurement and Neural Networks }\end{array}$ & 92.09 \\
\hline $\mathbf{2 0 1 8}$ & Proposed Technique & $\mathbf{9 5}$ \\
\hline
\end{tabular}

Table 6 shows that the recognition rate of proposed ASL numbers gestures recognition technique and recognition rate of the existing traditional ASL numbers gestures recognition techniques and with their recognition techniques used for ASL numbers gestures recognition.

A comparative recognition rate of existing traditional ASL numbers gestures recognition techniques and proposed ASL numbers gestures recognition method for recognizing gestures of ASL numbers is shown in Fig. 25

Table 6 and Fig. 25 clearly highlights the proposed ASL numbers gestures recognition method yields the better ASL numbers gestures recognition rate of $95 \%$ comparing with all the other existing traditional ASL numbers gestures recognition techniques. It is also noticed that the proposed ASL numbers gestures recognition method shows the huge recognition rate 
difference with the ASL numbers gestures recognition technique with recognition rate of $74.69 \%$ which is implemented using Statistical Measures technique [15], and also with the ASL numbers gestures recognition technique with the recognition rate of $82.92 \%$ which is implemented using Orientation Histogram technique [15]

\section{Comparitive Recognition Rate}
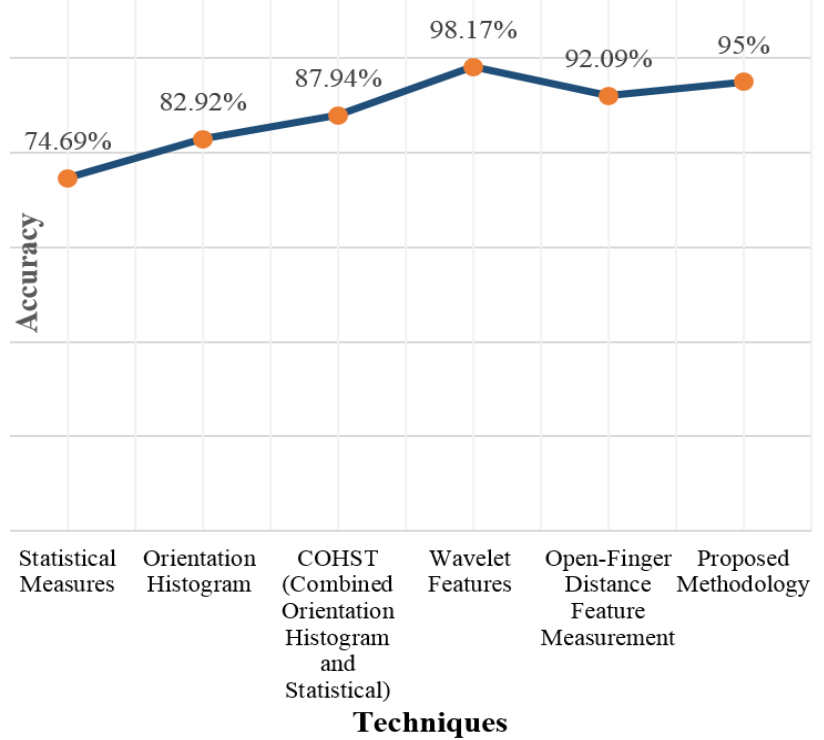

Fig.25. A Comparative Success Rate of existing techniques and proposed methodology for recognizing gestures of ASL Numbers.

Table 7 and Fig. 26 highlights the overall average recognition rate of Occluded Gestures of ASL Alphabets and Occluded Gestures of ASL Numbers. With respect to Occluded gestures, comparatively, Occluded ASL numbers gestures provides the satisfactory average recognition rate of $85 \%$ whereas occluded ASL alphabets gestures offers an average recognition rate of $70.83 \%$.

Table 7. Overall Average Recognition Rate of ASL Alphabets (Occluded) and ASL Numbers (Occluded)

\begin{tabular}{|l|c|}
\hline \multicolumn{1}{|c|}{ Occluded ASL Gestures } & Avg. Recognition Rate (in \%) \\
\hline ASL Alphabets & 70.83 \\
\hline ASL Numbers & 85 \\
\hline
\end{tabular}

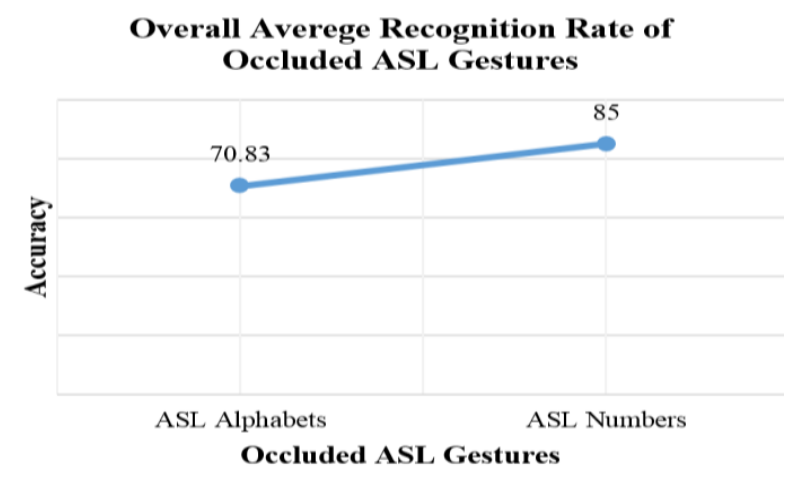

Fig.26. Overall Average Recognition Rate of Occluded Gestures (ASL Alphabets and ASL Numbers)
In Table 8 and Fig. 27, an overall average recognition rate of Non-Occluded Gestures of ASL Alphabets and Non-Occluded Gestures of ASL Numbers are highlighted. In consideration of non-occluded gestures, relatively, non-occluded ASL numbers gestures delivers an outstanding average recognition rate of $100 \%$ whereas non-occluded ASL alphabets gestures offers a better average recognition rate of $97.5 \%$.

Table 8. Overall Average Recognition Rate of ASL Alphabets (NonOccluded) and ASL Numbers (Non-Occluded).

\begin{tabular}{|c|c|}
\hline Non-Occluded ASL Gestures & Avg. Recognition Rate (in \%) \\
\hline ASL Alphabets & 97.5 \\
\hline ASL Numbers & 100 \\
\hline
\end{tabular}

Overall Average Recognition Rate of Non-Occluded ASL Gestures

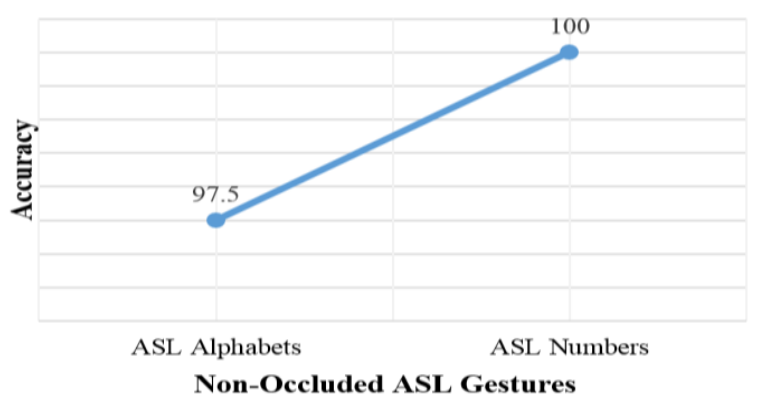

Fig.27. Overall Average Recognition Rate of Non-Occluded Gestures (ASL Alphabets and ASL Numbers)

An overall average recognition rate of Occluded ASL gestures (Both alphabets and Numbers are considered) and Non-Occluded ASL Gestures (Both alphabets and Numbers are considered) are highlighted in Table 9 and Figure 28. It is noticed in Table 9 and Figure 28, an overall average recognition rate of non-occluded ASL gestures (Both alphabets and Numbers are considered) is the better accuracy of $98.75 \%$ which is huge recognition rate than an overall average recognition rate of occluded ASL gestures (Both alphabets and Numbers are considered) of $77.21 \%$, which is less due to occluded gestures.

Table 9. Overall Average Recognition Rate of Occluded and NonOccluded Gestures

\begin{tabular}{|l|c|}
\hline \multicolumn{1}{|c|}{ ASL Gestures } & Avg. Recognition Rate (in \%) \\
\hline Occluded Gestures & 77.91 \\
\hline Non-Occluded Gestures & 98.75 \\
\hline
\end{tabular}

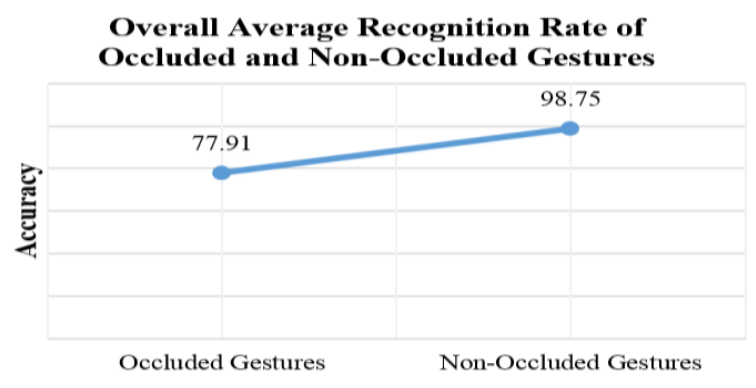

ASL Gestures

Fig.28. Overall Average Recognition Rate of Occluded and NonOccluded Gestures 
An overall average recognition rate ASL alphabets gestures (Both occluded and non-occluded gestures are considered) of 6 datasets of 6 different signers with different background colors and an overall average recognition rate ASL numbers gestures (Both occluded and non-occluded gestures are considered) of 6 datasets of 6 different signers with different background colors are emphasized in Table 10 and Fig. 29. Here, it is observed that the ASL numbers gestures (Both occluded and non-occluded gestures are considered) offers relatively better overall average recognition rate of $95 \%$ whereas the ASL alphabets gestures (Both occluded and non-occluded gestures are considered) offers $95 \%$ overall average recognition rate.

Table 10. Overall Average Recognition Rate of ASL Gestures

\begin{tabular}{|l|c|}
\hline \multicolumn{1}{|c|}{ ASL Gestures } & Avg. Recognition Rate (in \%) \\
\hline ASL Alphabets & $93.05 \%$ \\
\hline ASL Numbers & $95 \%$ \\
\hline
\end{tabular}

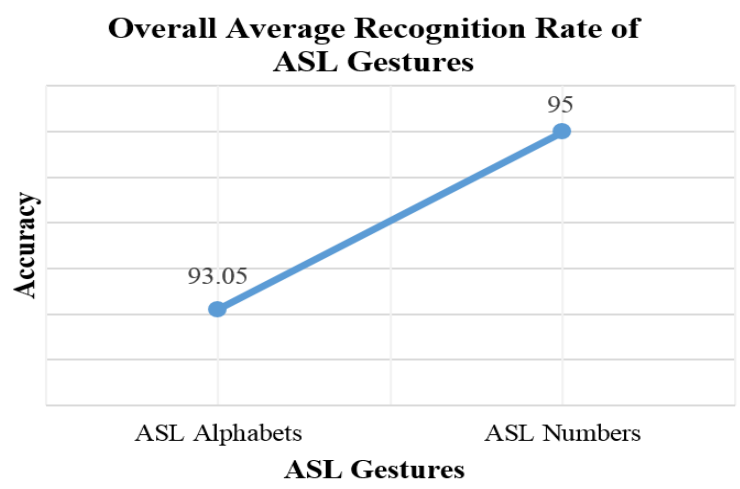

Fig.29. Overall Average Recognition Rate of ASL Gestures

\section{CONCLUSIONS}

This research paper exhibits an optimal approach, to accomplish the transliteration of 24 static ASL alphabets gestures (Letter $\mathbf{J}$ and $\mathrm{Z}$ have not included as they involve hand movement. Hence it requires video frames to be processed) and 10 static ASL numbers gestures into English text. Table 1 and 4 offers $93.05 \%$ and $95 \%$ of recognition rate of 6 sample sets of ASL Alphabets gestures and ASL Numbers gestures respectively. Table 2 and table 5 gives the statistical analysis of occluded and non-occluded gestures of ASL Alphabets gestures and ASL Numbers gestures respectively. Fig. 7, 8, 12, 13, 18, 19, 23, and 24 provides categorized recognition rates. Table 3 and Fig. 14 illustrates the result of our research work which proofs better recognition rate of ASL Alphabets gestures comparing with the existing traditional techniques. Table 6 and Fig. 25 illustrates the result of our research work which proofs the better recognition rate of ASL Numbers gestures comparing with existing techniques. Table 7, 8, 9, 10 and Fig. 26, 27, 28, 29 highlights the overall average recognition rate of gestures of ASL Alphabets and ASL Numbers in occluded and non-occluded environment.

\section{FUTURE PERSPECTIVE}

This research work can be extended to recognize the rotation and distance invariant ASL Alphabets gestures, numbers gestures and other complex gestures in different background (plain and complex), location (indoor and outdoor), lighting conditions (day and night light) in real time environment. This research work can also be extended to recognize English words and sentences which needs video processing.

\section{REFERENCES}

[1] B M Chethana Kumara, H S Nagendraswamy and R Lekha Chinmayi, "Spatial Relationship Based Features for Indian Sign Language Recognition", International Journal of Computing, Communications \& Instrumentation Engineering, Vol. 3, Issue 2, ISSN 2349. 1469,2016

[2] Srinath S, Ganesh Krishna Sharma, "Classification approach for Sign Language Recognition", International Conference on Signal, Image Processing, Communication \& Automation, 2017.

[3] Shivashankara S, Srinath S, "A comparative Study of Various Techniques and Outcomes of Recognizing American Sign Language: A Review", International Journal of Scientific Research Engineering \& Technology (IJSRET), Vol. 6, Issue 9, pp.1013-1023, 2017.

[4] Shivashankara S, Srinath S, "A Review on Vision Based American Sign Language Recognition, its Techniques, and Outcomes", $7^{\text {th }}$ IEEE International Conference on Communication Systems and Network Technologies (CSNT-2017), pp.293-299, 2017.

[5] Helen Cooper, Brian Holt, Richard Bowden, "Sign Language Recognition",

[6] Dr. Roger Sapsford, Victor Jupp, "Data Collection and Analysis", $2^{\text {nd }}$ Edition, Sage Publishing Ltd, 2006.

[7] http://www.asluniversity.com

[8] http://www.asl.tc

[9] http://www.lifeprint.com

[10] https://www.youtube.com

[11] Jayshree R. Pansare, Maya Ingle, "Vision-Based Approach for American Sign Language Recognition Using Edge Orientation Histogram", International Conference on Image, Vision and Computing, pp.86-90, 2016.

[12] Matheesha Fernando, Janaka Wijayanayaka, "Low cost approach for Real Time Sign Language Recognition", $8^{\text {th }}$ International Conference on Industrial and Information Systems, pp.637-642, 2013.

[13] Nagaraj N. Bhat, Y V Venkatesh, Ujjwal Karn, Dhruva Vig, "Hand Gesture Recognition using Self Organizing Map for Human Computer Interaction", International Conference on Advances in Computing, Communications and Informatics, pp.734-738, 2013.

[14] Fahad Ullah, "American Sign Language Recognition System for Hearing Impaired People Using Cartesian Genetic Programming", $5^{\text {th }}$ International Conference on Automation, Robotics and Applications, pp.96-99, 2011.

[15] Asha Thalange, Dr. S. K. Dixit, "COHST and Wavelet Features Based Static ASL Numbers Recognition", $2^{\text {nd }}$ International Conference on Intelligent Computing, Communication \& Convergence (Elsevier), pp.455-460, 2016.

[16] Asha Thalange, Shantanu Dixit, "Effect of thinning extent on ASL number recognition using open-finger distance 
feature measurement technique", International Conference on Signal Processing And Communication Engineering Systems (SPACES), pp.39-43, 2015.

[17] Sharmila Konwar, Sagarika Borah, Dr.T Tuithung, "An American Sign Language Detection System using HSV Color Model and Edge Detection", IEEE International Conference on Communication and Signal Processing, pp.743-747, 2014.

[18] Sruthi Upendran, Thamizharasi. A, "American Sign Language Interpreter System for Deaf and Dumb Individuals", International Conference on Control, Instrumentation, Communication and Computational Technologies (ICCICCT), 2014.

[19] Aditi Kalsh, N.S. Garewal, "Sign Language Recognition for Deaf \& Dumb", International Journal of Advanced Research in Computer Science and Software Engineering, Vol 3, Issue 9, pp.103-106, 2013

[20] Sriparna Saha, Rimita Lahiri, Amit Konar, Atulya K. Nagar, "A Novel Approach To American Sign Language Recognition Using MAdaline Neural Network", IEEE Symposium Series on Computational Intelligence (SSCI), 2016

[21] Geetha M, Rohit Menon, Suranya Jayan, Raju James, Janardhan G.V.V, "Gesture Recognition for American Sign Language with Polygon Approximation", IEEE International Conference on Technology for Education, pp.241-245, 2011.

[22] Nachamai. M, "Alphabet Recognition of American Sign Language: A Hand Gesture Recognition Approach Using Sift Algorithm", International Journal of Artificial Intelligence \& Applications, Vol.4, No.1, pp.105-115, 2013.

[23] Davi Hirafuji Neiva, Cleber Zanchettin, "A Dynamic Gesture Recognition System to Translate Between Sign Languages in Complex Backgrounds", $5^{\text {th }}$ Brazilian Conference on Intelligent Systems, pp.421-426, 2016.

[24] Zhi-hua Chen, Jung-Tae Kim, Jianning Liang, Jing Zhang, and Yu-Bo Yuan, "Real-Time Hand Gesture Recognition Using Finger Segmentation", Hindawi Publishing Corporation, The Scientific World Journal, Vol 2014, Article ID 267872, pp.1-9, 2014.

[25] Suchin Adhan and Chuchart Pintavirooj, "Alphabetic Hand Sign Interpretation using Geometric Invariance", IEEE International Conference on Biomedical Engineering, 2014.

[26] Taehwan Kim, Karen Livescu, Gregory Shakhnarovich, American Sign Language Finger spelling Recognition with phonological feature-based tandem models, IEEE workshop on Spoken Language Technology, pp.119-124, 2012.

[27] Gururaj P Surampalli, Dayanand J, Dhananjay M, An Analysis of Skin Pixel Detection using Different Skin Color Extraction Techniques, International Journal of Computer Applications, Vol. 54, No. 17, pp.1-5, 2012.

[28] Rafael C Gonzalez, Richard E Woods, "Digital Image Processing", $2^{\text {nd }}$ Edition, Prentice Hall, 2008.

\section{Authors' Profiles}

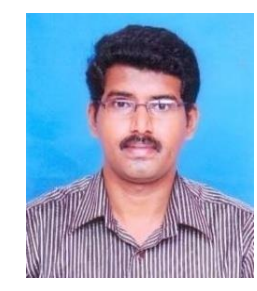

Shivashankara S, Research Scholar at Sri Jayachamarajendra College of Engineering, Mysuru (Visvesvaraya Technological University, Belagavi), India.

Born on April 29, 1980. B.E (2005), M.Tech (2008) from Visvesvaraya Technological University, Belagavi, India. Lecturer (2005), Asst. Prof. (2011) of Computer Science and Engineering.

He has published 3 scientific papers in international and national journals and conference proceedings. The main research interests include Image Processing and Pattern Recognition, and Machine Learning.

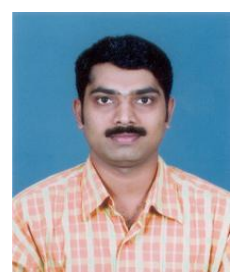

Dr.Srinath S Ph.D., Assistant Professor at JSS Science and Technology University, Mysuru, India. Received his Engineering degree in 1995, M.Tech ( $1^{\text {st }}$ Rank with Gold Medal) in 2002 and Ph.D. in 2015. He has published 18 scientific papers in international and national journals and conference proceedings. Executed 3 research projects sponsored from VTU, AICTE and MHRD (Government of India). Organized several training programs sponsored by different government agencies. Given more than 50 invited talks at different colleges. He has been the resource person for many training programs. The main research interests include Pattern Recognition and Image Processing.

How to cite this paper: Shivashankara S, Srinath S, " American Sign Language Recognition System: An Optimal Approach ", International Journal of Image, Graphics and Signal Processing(IJIGSP), Vol.10, No.8, pp. 18-30, 2018.DOI: $10.5815 /$ ijigsp.2018.08.03 